\title{
Ebola Virus Disease (EVD) and Women's Health Care in Pregnancy
}

\author{
Arash Khaki $^{1}$, Zhinous Bayatmakoo ${ }^{2}$
}

$\mathrm{E}$ bola virus (family Filoviridae, genus Ebolavirus, type species Zaire ebolavirus), Ebola hemorrhagic fever (EHF) or Ebola is a disease of human and other primates, caused by an Ebola virus (1-5). These agents cause a severe, unrelenting viral hemorrhagic fever with high mortality (1). EVD was first recognized in 1976, when two unrelated epidemics occurred in northern Zaire and southern Sudan (1-5). The largest outbreaks to date are the ongoing 2014 west African. Ebola outbreaks, which is affecting Guinea, Sierra Leone, Liberia and Nigeria (2-5).

\section{Transmission}

Ebola virus may be acquired upon contact with blood or bodily fluids of an infected animal. Spreading the air has not been documented in the natural environment. Fruit bats are believed to be a carrier and may spread the virus without being affected (1-2). Once human infection occurs, the disease may spread between people as well (2).

\section{Symptoms}

Clinical appearance is starts in 2 days to 3 weeks after contacting the virus, with fever, sore throat, muscle pain and headaches (1-5).

\section{Treatment}

A number of experimental treatment are being studied. The FDA has allowed two drugs, Zmapp and TKM-Ebola (2). Treatment is primarily supportive in nature (1-5). The disease has a high risk of death, killing between $50 \%$ and $90 \%$ of those infected with the virus (1-5), in conclusion No specific treatment for the disease is yet available.

\section{Prevention}

Includes decreasing the spread of disease from infected animals to humans (2). Prevention of epidemics rates on early recognition and initial cases and promp institution of barrier nursing. At the community level, properly sterilized injection equipment, protection from body fluid and skin during preparation of the dead, and routine barrier nursing precaution are probably adequate in most care (1-2). During the EHF epidemic in Kikwit, Democratic Republic of Congo the number of infected women was slightly higher than the man (6-7)

\section{Sex protection}

Saliva, breast milk, and semen, austerity from sex or the use of condoms during sex, as well as avoidance of breastfeeding and contact with the mucous membranes of the eye for at least 3 months after recovery, are still recommended to avoid possible exposure to EBOV in the aforementioned immunologically protected sites. Male survivors may be able to transmit the disease via semen for nearly two months (2).

\section{Pregnancy}

The number of infected women was slightly higher than the number of infected men.In research that done by Mupapa in 2000 confirmed, Among 105 women 15\% were pregnant and one of those survived. The mortality rate among pregnant woman with Ebola virus was slightly higher (93.3\%) than during the overall epidemic in the area (77\%). The mortality rate for non-pregnant women was $70 \%$ (6-7). Pregnant women might suffer hemorrhage due to various reasons such as Placenta Previa or abortion, making it difficult to diagnose Ebola in pregnant women. During an outbreaks of Ebola each expectation mother with bleeding receives care as a suspected Ebola patient, they may need blood transfusion and/or abortion and post-abortion care (6-7).

\section{Symptoms in Pregnancy}

Most clinical sign of ebola disease in pregnant women are: fever, asthenia, abdominal pain, conjunctivitis, anorexia, diarrhea, hiccups, arthralgia, shock, dysphagia, nausea, vomiting, retrosternal pain, cutaneous eruptio, increased respiratory rate. All infected women had with signs of hemorrhage, including severe genital bleeding, melena, gum bleeding, ecchymosis, bleeding at injection sites, hematemesis, petechia, and hematuria, respectively (7).

\section{Neuropsychiatric symptoms in pregnant women}

Headache, anxiety, decreased consciousness, apathy, coma, delirium, and convulsions, respectively (7).

\section{Abortion}

Spontaneous abortion is frequent. A high frequency of abortion has also been observed during infection. Previous study was confirmed that $(67 \%)$ of the pregnancy ended with an abortion in infected women with Ebola virus (7).

\section{References}

1. Peters CJ. Marburg and Ebola virus hemorrhagic fever. In: Mandel, Douglas, and Bennett's principles and practice of infectious disease. 7th ed. Philadelphia: Churchill Livingstone;2010. p 225-63.

2. Lyth N. Fragile economies in West Africa are being devastated by Ebola. Nurs Stand 2014;29(5):35.

3. Evans R. About Ebola app. Nurs Stand 2014;29(5):33.

4. Cheng AC, Kelly H. Are we prepared for Ebola and other viral haemorrhagic fevers? Aust N Z J Public Health 2014;38(5):403-4.

5. Vogel G. Infectious Diseases. Testing new Ebola tests. Science 2014;345(6204):1549-50

6. Twardowski L, McInnis T, Cappuccino CC, McDonald J, Rhodes J. Professional Responsibilities for Treatment of Patients with Ebola: Can a Healthcare Provider Refuse To Treat a Patient with Ebola? R I Med J (2013) 2014;97(10):63-64

7. Mupapa K, Mukundu W, Bwaka MA, Kipasa M, De Roo A, Kuvula $\mathrm{K}$, et al. Ebola hemorrhagic fever and pregnancy. J Infect Dis 1999;179 Suppl 1:S11-2. 